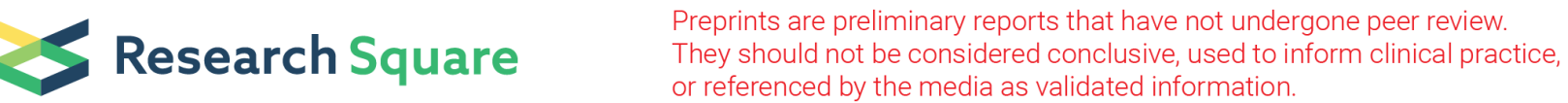

\section{In Vitro and in Vivo Tenocyte-protective Effectiveness of Dehydroepiandrosterone Against High Glucose-induced Oxidative Stress}

Shintaro Mukohara

Kobe University Graduate School of Medicine

Yutaka Mifune ( $\nabla$ m-ship@kf7.so-net.ne.jp)

Kobe University Graduate School of Medicine

Atsuyuki Inui

Kobe University Graduate School of Medicine

Hanako Nishimoto

Kobe University Graduate School of Medicine

Takashi Kurosawa

Kobe University Graduate School of Medicine

Kohei Yamaura

Kobe University Graduate School of Medicine

Tomoya Yoshikawa

Kobe University Graduate School of Medicine

Ryosuke Kuroda

Kobe University Graduate School of Medicine

\section{Research Article}

Keywords: High glucose, Oxidative stress, Dehydroepiandrosterone, NADPH oxidase, Diabetic tendinopathy

Posted Date: February 3rd, 2021

DOI: https://doi.org/10.21203/rs.3.rs-154654/v1

License: (c) (i) This work is licensed under a Creative Commons Attribution 4.0 International License. Read Full License 


\section{Abstract}

\section{Background}

Dehydroepiandrosterone (DHEA), an adrenal steroid, has a protective role against diabetes. The aim of this study was to investigate the in vitro and in vivo protective effects of DHEA against high glucoseinduced oxidative stress in tenocytes and tendons.

\section{Methods}

In an in vitro study, tenocytes from normal Sprague-Dawley rats were cultured in low-glucose (LG) or highglucose (HG) medium with or without DHEA. The experimental groups were: control group (LG without DHEA), LG with DHEA, HG without DHEA, and HG with DHEA. Reactive oxygen species (ROS) production, apoptosis, and messenger RNA (mRNA) expression of NADPH oxidase (NOX) 1 and 4, and interleukin-6 (IL-6) were determined. In the in vivo study, diabetic rats were divided into a control group and a DHEAinjected group (DHEA group). NOX1 and NOX4 protein expression and mRNA expression of NOX1, NOX4, IL-6, matrix metalloproteinase (MMP)-2, tissue inhibitors of matrix metalloproteinase (TIMP)-2, and type I and III collagens in the Achilles tendon were determined.

\section{Results}

In rat tenocytes, DHEA decreased the expression of NOX1 and IL-6, ROS accumulation, and apoptotic cells. In the diabetic rat Achilles tendon, NOX1 protein expression and mRNA expression of NOX1, IL-6, MMP-2, TIMP-2, and type III collagen were significantly lower, while type I collagen expression was significantly lower in the DHEA group.

\section{Conclusions}

DHEA showed antioxidant and anti-inflammatory effects both in vitro and in vivo. Moreover, DHEA improved tendon matrix synthesis and turnover which are affected by hyperglycemic conditions. DHEA could be a preventive drug for the diabetic tendinopathy.

\section{Background}

Musculoskeletal disorders such as tendinitis ${ }^{1}$, Dupuytren's disease ${ }^{2}$, carpal tunnel syndrome ${ }^{3}$, adhesive capsulitis $^{4}$ and calcific tendinopathy ${ }^{5}$ are more common in patients with diabetes mellitus (DM). DM is an important risk/causative factor associated with the development and poor prognosis of chronic tendinopathy ${ }^{6,7}$. It is reported that the potential mechanisms by which DM causes and exacerbates tendinopathy are: increase in cell death, reactive oxygen species (ROS), and pro-inflammatory cytokines, imbalance in the ratio of matrix metalloproteinase (MMP)/tissue inhibitors of MMP (TIMP), and impaired angiogenesis and tenogenic differentiation ${ }^{6}$. However, the pathogenic mechanisms of diabetic tendinopathy are not fully understood and there is no effective treatment for chronic tendinopathy. 
A number of studies have demonstrated that hyperglycemic conditions induce oxidative stress and cytokine production, leading to inflammation and tissue damage in various organs ${ }^{8,9}$. Under high glucose conditions, oxidative stress causes increased ROS production, which leads to cell death and tissue damage in a variety of cell types ${ }^{8,10,11}$. The main pathways of ROS production in hyperglycemia have been reported, such as NADPH oxidase (NOX) ${ }^{12}$, the accumulation of advanced glycation end-products ${ }^{13}$, and the increase in superoxide production by the mitochondrial electron transfer system ${ }^{14}$.

NOX is a family of multi-subunit enzymes located on the cell membrane and activated by protein kinase $\mathrm{C}$, causing ROS production ${ }^{12}$. Under pathological conditions, the upregulation of tissue- and diseasespecific NOX subtypes can cause overproduction of ROS ${ }^{15}$. Previous reports showed NOX1 expression was increased in rat tenocytes and Achilles tendons under hyperglycemic conditions, and the accompanying ROS overproduction and inflammatory reaction contributed to diabetic tendinopathy ${ }^{16,17}$.

Dehydroepiandrosterone (DHEA) is the most abundant circulating steroid hormone produced by the mammalian adrenal cortex ${ }^{18}$. The natural concentration of DHEA is around $10 \mu \mathrm{M}$ in young adults but decreases with age ${ }^{19}$. This decline in DHEA suggests that a relative deficiency of this steroid may be causally related to the development of age-related illnesses such as atherosclerosis, metabolic diseases, and cancer ${ }^{20,21}$. In fact, there are numerous reports on the protective effects of DHEA in reducing antioxidant species and restoring antioxidant defense systems, including in the liver, kidney, muscle ${ }^{22}$, and serum of humans with type 2 diabetes $^{23}$. However, the antioxidant effects of DHEA on tenocytes and tendons are still unknown. Here, we aimed to evaluate the in vitro and in vivo effects of DHEA on high glucose-induced oxidative stress in tenocytes and tendons.

\section{Methods}

All animal procedures were performed with the approval and guidance of the Animal Care and Use Committee of our institution. The experiments were conducted in accordance with the ARRIVE guidelines.

\section{In vitro experiments: cell preparation}

Achilles tendons were excised from 15 healthy male Sprague-Dawley (SD) rats of eight weeks of age. Tendons were washed twice with phosphate-buffered saline (PBS) and cut into small pieces measuring approximately 1.5 to $2.0 \mathrm{~mm}^{3}$. Several pieces were placed on a culture plate and cultured in Dulbecco's modified Eagle's medium (DMEM) (Sigma-Aldrich, St. Louis, MO, USA) supplemented with $10 \%$ fetal bovine serum (FBS), $100 \mu \mathrm{g} / \mathrm{mL}$ streptomycin, and $100 \mathrm{U} / \mathrm{mL}$ penicillin. The explants were incubated at $37{ }^{\circ} \mathrm{C}$ in a humidified atmosphere of $5 \% \mathrm{CO}_{2} / 95 \%$ air. After the tenocytes from explants had attained a subconfluent state, the cells were subcultured after trypsin digestion. The culture medium was changed every five days. Cells from passages 2 to 3 were used in this study. 


\section{In vitro experiments: cell proliferation assays}

Cell proliferation was measured by a water-soluble tetrazolium salt (WST) assay using a Cell Counting Kit-8 (Dojindo, Kumamoto, Japan). A total of 5000 cells were seeded in $100 \mu \mathrm{L}$ of DMEM in each well of a 96-well plate. Cells were cultured for 24 hours in a $\mathrm{CO}_{2}$ incubator at $37{ }^{\circ} \mathrm{C}$ before the WST assay evaluation and then incubated for another 48 hours in DMEM containing four different DHEA (Tokyo Chemical Industry, Tokyo, Japan) concentrations ( 0 as a control, 1, 10, 20, and $50 \mu \mathrm{M}$ ). DHEA was dissolved in $0.1 \%$ dimethyl sulfoxide (DMSO). For the WST assay, each well was supplemented with 10 $\mu \mathrm{L}$ of WST for four hours at $37^{\circ} \mathrm{C}$ in a $\mathrm{CO}_{2}$ incubator before spectrophotometric evaluation at $450 \mathrm{~nm}$ ( $n=15$ per group).

\section{In vitro experiments囚experimental protocol}

Tenocytes were seeded onto 12-well culture plates at $10^{5}$ cells per well and incubated in DMEM with two different glucose concentrations: $6 \mathrm{mM}$ in the low-glucose (LG) group, and $33 \mathrm{mM}$ in the high-glucose $(\mathrm{HG})$ group according to a previous study ${ }^{24}$. DHEA was dissolved in DMSO to obtain a $2 \mathrm{mM}$ stock solution and diluted to a final concentration of $10 \mu \mathrm{M}^{19}$. DHEA was added at cell seeding and the tenocytes were divided into four groups: the control group (LG DHEA-), LG with DHEA (LG DHEA+), HG without DHEA (HG DHEA-), and HG with DHEA (HG DHEA+) ( $n=12$ per group). The same amount of DMSO was added to all groups ( $n=15$ per group).

\section{In vitro experiments: Quantitative real-time polymerase chain reaction (PCR) analysis}

At 48 hours, total RNA from tenocytes was extracted using a RNeasy Mini Kit (Qiagen, Valencia, CA, USA). Using a High Capacity cDNA Reverse Transcription Kit (Applied Biosystems, Foster City, CA, USA), total RNA was reverse-transcribed into single-strand complementary DNA (cDNA). PCR in triplicate was performed on the CDNA with 7900HT Fast Real-Time PCR System and SYBR Green reagents (Applied Biosystems). The messenger RNA (mRNA) levels of NOX1, NOX4, and IL-6 were analyzed. The list of primer sequences are shown in Table 1. Results were normalized to the mRNA levels of the housekeeping gene glyceraldehyde 3-phosphate dehydrogenase (GAPDH) and expressed relative to their levels in the control culture using the $2^{-\Delta \Delta C t}$ method ${ }^{25}$ as previous studies ${ }^{16,17}$ ( $n=15$ per group). 
Table 1

Primer sequences used for polymerase chain reaction.

\begin{tabular}{|c|c|}
\hline Gene & Oligonucleotide sequence \\
\hline NOX1 & $\begin{array}{l}\text { Forward 5' GTGGCTTTGGTTCTCATGGT 3' Reverse 5' TGAGGACTCCTGCAACTCCT } \\
3^{\prime}\end{array}$ \\
\hline NOX4 & $\begin{array}{l}\text { Forward 5' GGGCCTAGGATTGTGTTTGA 3' Reverse 5' CTGAGAAGTTCAGGGCGTTC } \\
3^{\prime}\end{array}$ \\
\hline $\begin{array}{l}\text { Type } \otimes \\
\text { collagen }\end{array}$ & Forward 5' TGGAGACAGGTCAGACCTG 3' Reverse 5' TATTCGATGACTGTCTTGCC 3' \\
\hline $\begin{array}{l}\text { Type } \mathbb{Q} \\
\text { collagen }\end{array}$ & $\begin{array}{l}\text { Forward 5' TAAAGGGTGAACGGGGCAGT 3' Reverse 5' ACGTTCCCCATTATGGCCAC } \\
3^{\prime}\end{array}$ \\
\hline MMP-2 & 'Forward 5' GGAAGCATCAAATCGGACTG 3' Reverse 5' GGGCGGGAGAAAGTAGCA 3' \\
\hline TIMP-1 & $\begin{array}{l}\text { Forward 5' ATAGTGCTGGCTGTGGGGTGTG 3' Reverse 5' } \\
\text { TGATCGCTCTGGTAGCCCTTCTC 3' }\end{array}$ \\
\hline TIMP-2 & $\begin{array}{l}\text { Forward 5' GGACACGCTTAGCATCACCCAGA 3' Reverse 5' } \\
\text { GTCCATCCAGAGGCACTCATCC 3' }\end{array}$ \\
\hline IL-6 & $\begin{array}{l}\text { Forward 5' GGTCTTCTGGAGTTCCGTTTC 3' Reverse 5' } \\
\text { GGTCTTGGTCCTTAGCCATCT 3' }\end{array}$ \\
\hline GAPDH & $\begin{array}{l}\text { Forward 5' GGTGGTCTCCTCTGACTTCAACA 3' Reverse 5' } \\
\text { GTTGCTGTAGCCAAATTCGTTGT 3' }\end{array}$ \\
\hline
\end{tabular}

\section{In vitro experiments: detection of ROS accumulation}

According to previous reports ${ }^{16,17}$, intracellular ROS levels in tenocytes of each group were detected by the oxidation-sensitive fluorescent probe dichloro-dihydro-fluorescein diacetate (DCFH-DA) using the Total ROS/Superoxide Detection Kit (Enzo Life Science, Farmingdale, NY, USA) in accordance with the manufacturer's protocol. Tenocytes $\left(5 \times 10^{4}\right)$ were incubated with DCFH-DA at a final concentration of 10 $\mu \mathrm{M}$ for 60 minutes at $37{ }^{\circ} \mathrm{C}$ in the dark, washed three times with PBS, trypsinized, and resuspended. For quantification, the number of ROS-positive cells and DAPI-positive cells in four rectangular areas $(0.75$ $\mathrm{mm} \times 1.0 \mathrm{~mm}$ ) in each slide were counted and the mean values were calculated. The percentage of ROSpositive cells was calculated the formula (number of ROS-positive nuclei/number of DAPI-positive nuclei) $\times 100$ and expressed as the mean of the four areas ( $n=15$ per group).

\section{In vitro experiments: Immunofluorescence staining for analysis of apoptotic cells}

According to previous studies ${ }^{16,17}$, nuclear fragmentation was detected by terminal deoxynucleotidyl transferase dUTP nick end labelling (TUNEL) staining with an APO-DIRECT Kit (Phoenix Flow Systems, 
San Diego, CA, USA) according to the manufacturer's protocol, using fixed cells ( $4 \%$ paraformaldehyde/PBS) with 2-(4-amidinophenyl)-1H-indole-6-carboxamidine (DAPI). For quantification, the number of apoptosis-positive and DAPI-positive cells in four rectangular areas $(0.75 \mathrm{~mm} \times 1.0 \mathrm{~mm})$ in each slide were counted and the mean values were calculated. The percentage of apoptosis-positive cells was calculated using the formula (number of apoptosis-positive nuclei/number of DAPI-positive nuclei) $\times$ 100 and expressed as a mean of the four areas ( $n=15$ per group).

\section{In vivo animal experiments: type I diabetic rat model}

To induce DM, a single dose of streptozotocin (STZ; 65 mg/kg; Sigma-Aldrich) dissolved in sodium citrate buffer ( $\mathrm{pH} 4.5)$ was intravenously injected into 18 eight-week-old healthy male SD ${ }^{26}$. Following the injections, all animals were housed in standard cages with unrestricted food, water, and activity. All STZinjected rats became diabetic 10 days after STZ injection. Their mean blood glucose level was $405.8 \pm$ $62.5 \mathrm{mg} / \mathrm{ml}$ (mean \pm standard deviation), although that of healthy control rats was $<150 \mathrm{mg} / \mathrm{dl}^{26}$.

\section{In vivo animal experiments: experimental protocol}

DM rats were randomly divided into 2 groups: a control group and a DHEA-injected group (DHEA group) ( $\mathrm{n}=9$ in each group). In the DHEA group, two weeks after STZ injection, $50 \mathrm{mg} / \mathrm{kg} \mathrm{DHEA}^{27,28}$ and vehicle (10\% DMSO) were injected intraperitoneally every other day for 4 weeks. In the control group, vehicle alone was injected in the same manner. The animals were sacrificed 4 weeks after the first DHEA or vehicle injection (6weeks after STZ injection) according to a previous study ${ }^{16}$. The right Achilles tendon was used for immunohistological evaluation, and the left was used for quantitative real-time PCR.

\section{In vivo animal experiments: quantitative real-time PCR analysis}

Nine left tendons in each of DHEA group and control group were used for quantitative real-time PCR. The Achilles tendons were cut into small pieces and minced. Isolated Achilles tendons were enzymatically dissociated with type II collagenase (Worthington Biochemical Corporation, Lakewood, NJ, USA) and prepared for RNA isolation ${ }^{25}$. Total RNA was extracted using a RNeasy Mini Kit. Reverse transcription into single-stranded CDNA and real-time PCR was performed as previously described. Expression of NOX1, NOX4, IL-6, MMP-2, TIMP-2, and type I and III collagen (col1 and col3) was evaluated as previously described. 


\section{In vivo animal experiments: Achilles tendon histology and immunohistochemistry for NOX analysis}

For immunohistological analysis, hematoxylin and eosin (H\&E) staining, and staining for NOX1 and NOX4 were performed using the nine right tendons of diabetic rats from each group according to previous study ${ }^{16}$. Frozen, long-axis sections of Achilles tendons were sectioned into $7 \mu \mathrm{m}$ thick specimens and fixed by using $10 \%$ phosphate-buffered paraformaldehyde at room temperature. Histological evaluation of fiber structure and arrangement, nuclear morphology, and zonal variations in tendon cellularity was performed using H\&E staining ${ }^{29}$. Each variable was scored between 0 and 3; 0 being normal, 1 slightly abnormal, 2 abnormal, and 3 markedly abnormal ${ }^{29}$. The grading of H\&E-stained sections was performed in five randomly selected optical fields in each section and evaluated by two blinded investigators.

The immunohistochemical evaluation of NOX expression was performed by using anti-NOX1 and antiNOX4 antibodies (Abcam, Cambridge, UK). Sections were incubated with proteinase for 10 minutes, treated with $3 \%$ hydrogen peroxide (Wako Pure Chemical Industries, Osaka, Japan) to block endogenous peroxidase activity, and incubated with anti-NOX1 or anti-NOX4 antibodies (1:100 for both) at $4{ }^{\circ} \mathrm{C}$ overnight ${ }^{16}$. Then, sections were incubated with a peroxidase-labeled immunoglobulin antibody (Nichirei Bioscience, Tokyo, Japan) at room temperature for 30 minutes $^{16}$. The signal (NOX1 and NOX4) was detected by the formation of a brown color following incubation with the peroxidase substrate 3,3'diaminobenzidine (Nichirei Bioscience). Sections were counterstained with hematoxylin and examined microscopically. For semi-quantitative analysis, the ratio of NOX-positive tendon cells per field was determined in five randomly selected fields for each tissue section ${ }^{16}$.

\section{Statistical analysis}

All data are expressed as means and standard deviations. All statistical analyses of recorded data were performed using the Excel statistical software package (Ekuseru-Toukei 2015; Social Survey Research Information Co., Ltd., Tokyo, Japan). Comparisons between more than two groups were performed by analysis of variance and Tukey's post hoc test. Comparisons between two groups were performed using analysis of variance and the Mann-Whitney $U$ test. A p-value $<0.05$ was considered statistically significant.

\section{Results}

\section{In vitro experiments: cell proliferation assays}

The WST assay of tendon cell proliferation showed that the 1, 10, and $20 \mu \mathrm{M}$ DHEA groups had significantly enhanced cell viability compared to the control group $(p<0.05)$, and the viability of the $10 \mu \mathrm{M}$ DHEA group was the highest among all groups. The 20 and $50 \mu \mathrm{M}$ DHEA groups were lower in a dose-dependent manner (Fig. 1). 


\section{In vitro experiments: Quantitative real-time PCR analysis}

At 48 hours, NOX1 and IL-6 mRNA expression in the HG DHEA- group was significantly higher than in the LG DHEA-group $(p<0.05)$. NOX1 and IL-6 mRNA expression in the HG DHEA+ group was significantly lower than in the HG DHEA-group $(p<0.05)$. There was no significant difference in the expression of NOX4 mRNA within each group (Fig. 2).

\section{In vitro experiments: ROS accumulation}

The cytoplasm of ROS-positive cells was stained green (Fig.3a). A quantitative analysis of ROS-positive cells is shown in Figure 3b. The ROS accumulation in the HG DHEA-group was significantly greater than that of the LG DHEA-group $(p<0.05)$. Accumulation in the HG DHEA+ group was significantly lower than that of the HG DHEA- group ( $p<0.05)$. There was no difference between the LG groups (Fig.3b).

\section{In vitro experiments: Immunofluorescence staining for analysis of apoptotic cells}

Apoptotic cells were observed in both HG groups, and abnormal nuclear morphology such as nuclear fragmentation was found in apoptotic cells (Fig.4a). A quantitative analysis of apoptotic cells is shown in Figure 4b. The number of apoptotic cells in the HG DHEA+ group was lower than that in the HG DHEAgroup, and there was a statistically significant difference between the two groups $(p<0.05)($ Fig.4b).

\section{In vivo animal experiments: Achilles tendon histology and immunohistochemistry for NOX analysis}

Histological evaluation showed no significant difference in fiber structure and arrangement, rounding of the nuclei, and regional variations in cellularity between the control and DHEA groups (Table 2). The fiber structure and arrangement showed near parallel collagen fiber orientation and flattened or spindle-shaped nuclei arranged in rows between the collagen fibers in both groups (Fig.5).

Immunohistochemical staining of the Achilles tendon at four weeks following DHEA injection showed that NOX1 expression markedly decreased within the tenocytes of diabetic rats with DHEA (Fig.6a). NOX4 was weakly expressed in both groups and showed no difference between the two groups (Fig. 6a). Using semi-quantitative analysis, the percentage of NOX1-positive cells was significantly lower in the Achilles tendon of the DHEA group ( $<0.05)$ (Fig.6b). There was no significant difference in the percentage of NOX4-positive cells between the groups $(p<0.05)$ (Fig.6b). 
Table 2

Tendon pathological scores.

\begin{tabular}{|llll|}
\hline & Mean control (SD) & Mean DHEA (SD) & p-value * \\
\hline Fibre struture & $0.89(0.60)$ & $0.74(0.57)$ & 0.22 \\
\hline Fibre arrangement & $0.91(0.54)$ & $0.83(0.60)$ & 0.44 \\
\hline Nuclear morphological changes (rounding) & $0.67(0.59)$ & $0.52(0.50)$ & 0.25 \\
\hline Regional variations in cellularity & $0.45(0.49)$ & $0.39(0.49)$ & 0.52 \\
\hline * Mann-Whitney U test. & & & \\
$\mathrm{n}=9$ rats in the control group, $\mathrm{n}=9$ rats in the DHEA group. & & \\
\hline
\end{tabular}

\section{In vivo animal experiments: quantitative real-time PCR}

The expression of NOX1 in Achilles tendons was significantly lower in the DHEA group $(p<0.05)$. There was no significant difference in NOX4 expression between the control and DHEA groups (Fig. 7). The expression of IL-6, MMP-2, TIMP-2, and col3 was significantly lower in the DHEA group $(p<0.05)$, while the expression of col1 was significantly higher in the DHEA group $(p<0.05)$ (Fig.7).

\section{Discussion}

DHEA is a circulating steroid hormone abundantly produced by the mammalian adrenal cortex ${ }^{18}$. DHEA has the potential to improve the disease status of conditions such as DM, osteoporosis, arterial sclerosis, Alzheimer's disease, and adrenal insufficiency due to its antioxidant effects ${ }^{14,15}$. The antioxidant effects of DHEA have been reported to be mediated by the activation of the Nrf2 pathway ${ }^{19}$ and inhibition of glucose-6-phosphate dehydrogenase (G6PD) ${ }^{30}$, for example; however, the mechanistic details are not fully understood. In the field of orthopedics, DHEA has been shown to exert beneficial effects on osteoarthritis-damaged cartilage by actively regulating the balance of anabolic and catabolic factors (e.g., MMPs/TIMP-1 and ADAMTS/TIMP-3) and inhibiting catabolic signaling pathways (e.g., Wnt/ $\beta$ catenin $)^{31}$. Although there are many studies regarding the effects of DHEA on knee osteoarthritis, there are no reports concerning the effectiveness of DHEA in preventing and treating tendon disease.

High glucose conditions upregulate the expression of NOX1 mRNA and ROS production in rat tenocytes and Achilles tendon ${ }^{16,17}$ suggesting that overexpression of NOX may be a therapeutic target for diabetic tendinopathy. Here, the in vitro effectiveness of DHEA on hyperglycemia-induced oxidative stress in rat tenocytes was investigated. DHEA enhanced the viability of tenocytes and had no cytotoxic effects as reported in muscle cells ${ }^{19}$ and it suppressed the expression of NOX1, the overproduction of ROS, and 
apoptosis. Additionally, DHEA decreased NOX1 expression (mRNA and protein) in the diabetic rat Achilles tendon.

Huerta-García et al. demonstrated the protective effects of DHEA against oxidative stress induced by high concentrations of glucose in endothelial cells ${ }^{32}$. Kiersztan et al. reported that DHEA reduced oxidative stress in the kidney-cortex due to a decline in NOX activity ${ }^{33}$, and it was considered that DHEA exerted an antioxidant effect by a same mechanism in tenocytes. Although the mechanism of action of DHEA on tenocytes has not been fully elucidated in this study, DHEA showed antioxidant effects on high glucoseinduced oxidative stress through suppression of NOX1 expression. This suggests that DHEA may be effective as a preventive medicine for diabetic tendon disorders.

It was reported that no significant change was observed in the histological evaluation of diabetic rat Achilles tendon at 6 weeks after STZ administration compared with normal SD rats ${ }^{16,34}$, and similar results were obtained in this study. These results were thought to be due to the evaluation of the initial response of diabetic tendinopathy ${ }^{16,34}$.

A systematic review of cytokines in tendon disease reported that the expression of IL-1 $\beta$, IL- 6 , and TNF-a in animal tendon injury models tended to increase from the early phase of tendon healing ${ }^{16}$. IL- 6 was the only cytokine involved in human tendon disease and elevated in tendon tears ${ }^{35}$. A previous study showed a higher expression of IL-6 in rat tenocytes and Achilles tendon under high-glucose conditions and indicated that high-glucose conditions might stimulate inflammatory processes within the tendon ${ }^{16}$. Additionally, type III collagen is expressed during inflammatory processes, although approximately $90 \%$ of the collagen in normal tendons is type $\mathrm{I}^{36}$. Our in vitro and in vivo studies showed that DHEA had antiinflammatory effects in decreasing IL-6 expression in rat tenocytes and Achilles tendons, and decreased the expression of type III collagen while increasing type I collagen expression.

The balance between MMP and TIMP expression regulates normal tendon metabolic activity ${ }^{37}$. During inflammation, MMPs cleave damaged interstitial collagen for remodeling, while TIMPs inhibit the overexpression of MMPs ${ }^{37}$. MMP-2 and TIMP-2 are increased in the diabetic rat Achilles tendon ${ }^{16}$. In the present study, DHEA significantly decreased the expression of MMP-2 and TIMP-2 in the Achilles tendon of diabetic rats. It is possible that DHEA regulates the balance of MMP-2/TIMP-2 and improves tendon matrix synthesis and turnover.

This study had several limitations. First, the monolayer culture of tenocytes in vitro does not reproduce true physiological conditions. However, previous studies have demonstrated that primary tenocytes maintained phenotypical stability until passage 5 when passaged in subconfluence ${ }^{38,39}$. Isolation of cells from the native tendon was performed according to previously reported and accepted methods ${ }^{40}$. Second, our in vivo experiments have evaluated early changes in diabetic tendinopathy, thus there was no changes in tendon mechanical properties or fibril organization. Further research is needed on the longterm effects of DHEA. Third, the biomechanical properties of DHEA on the Achilles tendon in diabetic rats were not investigated due to low experimental numbers. Fourth, our results indicated that DHEA has 
antioxidant and anti-inflammatory properties in tenocytes and tendons under hyperglycemic conditions. However, the clinical efficacy of DHEA supplementation in humans to reduce patho-physiological symptoms related to diabetic tendinopathy remains to be evaluated.

\section{Conclusions}

DHEA showed antioxidant and anti-inflammatory effects by reduction in the expression of NOX1 and IL-6, the overproduction of ROS and the suppression of apoptosis in our experimental model. Additionally, DHEA improved tendon matrix synthesis and turn over by reducing the expression of MMP-2/TIMP-2 and type III collagen induced by hyperglycemic conditions. These results suggest that DHEA could be a preventive drug for the diabetic tendinopathy.

\section{Abbreviations}

DM: Diabetes mellitus, ROS: reactive oxygen species, NOX: NADPH oxidase

MMP: matrix metalloproteinase, TIMP: tissue inhibitors of matrix metalloproteinase

DHEA: Dehydroepiandrosteron

SD: Sprague-Dawley, PBS; phosphate buffered saline

DMEM: Dulbecco's Modified Eagle's Medium, FBS: fetal bovine serum

PCR: polymerase chain reaction, STZ: streptozotocin, DMSO: dimethyl sulfoxide

H\&E: hematoxylin and eosin

\section{Declarations}

\section{Ethics approval consent to participate}

Our IRB (Institutional Animal Care and Use Committee At Kobe University) provided the approval for our study and the approval informations are follows. (Permission Number: P190208). All animal procedures were performed under the approval and guidance of our IRB.

\section{Consent to participate}

Not applicable.

\section{Consent for publication}




\section{Availability of data and materials}

The datasets generated during and analyzed during the current study are not publicly available due to the inclusion of unpublished data but are available from the corresponding author on reasonable request.

\section{Competing interests}

The authors declare that they have no competing interests.

\section{Funding}

Funding information is not applicable.

\section{Authors' contributions}

YM and Al contributed to the conception and design of the study. TKU, KY and TY performed the experiments and collected the data. Data and statistical analysis were done by YM and Al. Manuscript preparation was done by YM and Al. Supervising was done by RK. All authors read and approved the final manuscript.

\section{Acknowledgements}

The authors would like to give a special thanks to Ms. MYasuda, Ms. KTanaka and Ms. MNagata (Department of Orthopaedic Surgery, Kobe University Graduate School of Medicine) for their excellent technical assistance. The authors would like to thank Editage (www.editage.com) for English language editing.

\section{References}

1. Yosipovitch G, Yosipovitch Z, Karp M, Mukamel M. Trigger finger in young patients with insulin dependent diabetes. J Rheumatol 1990;17:951-952.

2. Noble J, Heathcote JG, Cohen H. Diabetes mellitus in the aetiology of Dupuytren's disease. J Bone Joint Surg [Br] 1984;66-B:322-325.

3. Pourmemari MH, Shiri R. Diabetes as a risk factor for carpal tunnel syndrome: a systematic review and meta-analysis. Diabet Med 2016;33:10-16. 
4. Balci N, Balci MK, Tüzüner S. Shoulder adhesive capsulitis and shoulder range of motion in type II diabetes mellitus: association with diabetic complications. J Diabetes Complications 1999;13:135140.

5. Mavrikakis ME, Drimis S, Kontoyannis DA, Rasidakis A, Moulopoulou ES, Kontoyannis S, et al. Calcific shoulder periarthritis (tendinitis) in adult onset diabetes mellitus: a controlled study. Ann Rheum Dis 1989;48:211-214.

6. Lui PPY. Tendinopathy in diabetes mellitus patients-Epidemiology, pathogenesis, and management. Scand J Med Sci Sports 2017;27:776-87.

7. Ranger TA, Wong AM, Cook JL, Gaida JE. Is there an association between tendinopathy and diabetes mellitus? A systematic review with meta-analysis. Br J Sports Med 2016;50:982-9.

8. Baynes JW, Thorpe SR. Role of oxidative stress in diabetic complications: a new perspective on an old paradigm. Diabetes 1999;48:1-9.

9. Nishikawa T, Edelstein D, Du XL, Yamagishi S, Matsumura T, Kaneda Y, et al. Normalizing mitochondrial superoxide production blocks three pathways of hyperglycaemic damage. Nature 2000;404:787-90.

10. Forbes JM, Coughlan MT, Cooper ME. Oxidative stress as a major culprit in kidney disease in diabetes. Diabetes 2008;57:1446-54.

11. Jansen F, Yang X, Franklin BS, Hoelscher M, Schmitz T, Bedorf J, et al. High glucose condition increases NADPH oxidase activity in endothelial microparticles that promote vascular inflammation. Cardiovasc Res 2013;98:94-106.

12. Inoguchi T, Li P, Umeda F, Yu HY, Kakimoto M, Imamura M, et al. High glucose level and free fatty acid stimulate reactive oxygen species production through protein kinase $\mathrm{C}$-dependent activation of NAD $(P) H$ oxidase in cultured vascular cells. Diabetes 2000;49:1939-45.

13. Goh SY, Cooper ME. Clinical review: the role of advanced glycation end products in progression and complications of diabetes. J Clin Endocrinol Metab 2008;93:1143-2.

14. Yu T, Jhun BS, Yoon Y. High-glucose stimulation increases reactive oxygen species production through the calcium and mitogen-activated protein kinase-mediated activation of mitochondrial fission. Antioxid Redox Signal 2011;14:425-37.

15. Manea SA, Constantin A, Manda G, Sasson S, Manea A. Regulation of Nox enzymes expression in vascular pathophysiology: focusing on transcription factors and epigenetic mechanisms. Redox Biol 2015;5:358-66.

16. Ueda Y, Inui A, Mifune Y, Sakata R, Muto T, Harada Y, et al. The effects of high glucose condition on rat tenocytes in vitro and rat Achilles tendon in vivo. Bone Joint Res 2018;7:362-72.

17. Kurosawa T, Mifune $Y$, Inui A, Nishimoto H, Ueda Y, Kataoka T, Yamaura K, et al. Evaluation of apocynin in vitro on high glucose-induced oxidative stress on tenocytes. Bone Joint Res 2020;9:23-8.

18. Labrie F. DHEA, important source of sex steroids in men and even more in women. Prog Brain Res 2010;182:97-148. 
19. Jeon S, Hur J, Kim J. DHEA Alleviates Oxidative Stress of Muscle Cells via Activation of Nrf2 Pathway. Appl Biochem Biotechnol 2015;176:22-32.

20. Chua CK, Henderson VW, Dennerstein L, Ames D, Szoeke C. Dehydroepiandrosterone sulfate and cognition in midlife, post-menopausal women. Neurobiol Aging 2014;35:1654-5.

21. Yoshida S, Aihara K, Azuma H, Uemoto R, Sumitomo-Ueda Y, Yagi S, et al. Dehydroepiandrosterone sulfate is inversely associated with sex-dependent diverse carotid atherosclerosis regardless of endothelial function. Atherosclerosis 2010;212:310-5.

22. Aragno M, Mastrocola R, Catalano MG, Brignardello E, Danni O, Boccuzzi G. Oxidative stress impairs skeletal muscle repair in diabetic rats. Diabetes 2004;53:1082-8.

23. Brignardello E, Runzo C, Aragno M, Catalano MG, Cassader M, Perin PC, Boccuzzi G. Dehydroepiandrosterone administration counteracts oxidative imbalance and advanced glycation end product formation in type 2 diabetic patients. Diabetes Care 2007;30:2922-7.

24. Portal-Núñez S, Ardura JA, Lozano D, Martínez de Toda I, De la Fuente M, Herrero-Beaumont G, et al. Parathyroid hormone-related protein exhibits antioxidant features in osteoblastic cells through its $\mathrm{N}$ terminal and osteostatin domains. Bone Joint Res 2018;7:58-68.

25. Livak KJ, Schmittgen TD. Analysis of relative gene expression data using real-time quantitative PCR and the 2(-Delta Delta C(T)) Method. Methods 2001;25:402-8.

26. Furman BL. Streptozotocin-induced diabetic models in mice and rats. Curr Protoc Pharmacol 2015;70:1-20.

27. Aksoy H, Yapanoglu T, Aksoy Y, Ozbey I, Turhan H, Gursan N. Dehydroepiandrosterone treatment attenuates reperfusion injury after testicular torsion and detorsion in rats. J Pediatr Surg 2007;42:1740-4

28. Pelissier MA, Trap C, Malewiak MI, Morfin R. Antioxidant effects of dehydroepiandrosterone and 7alpha-hydroxy-dehydroepiandrosterone in the rat colon, intestine and liver. Steroids 2004;69:137-44.

29. Maffulli N, Barrass V, Ewen SW. Light microscopic histology of achilles tendon ruptures. A comparison with unruptured tendons. Am J Sports Med 2000;28:857-63.

30. Handala L, Domange B, Ouled-Haddou H, Garçon L, Nguyen-Khac E, Helle F, et al. DHEA prevents ribavirin-induced anemia via inhibition of glucose-6-phosphate dehydrogenase. Antiviral Res 2017;146:153-60.

31. Huang K, Wu LD. Dehydroepiandrosterone: Molecular mechanisms and therapeutic implications in osteoarthritis. J Steroid Biochem Mol Biol 2018;183:27-38

32. Huerta-García E, Ventura-Gallegos JL, Victoriano ME, Montiél-Dávalos A, Tinoco-Jaramillo G, LópezMarure R. Dehydroepiandrosterone inhibits the activation and dysfunction of endothelial cells induced by high glucose concentration. Steroids 2012;77:233-40

33. Kiersztan A, Trojan N, Tempes A, Nalepa P, Sitek J, Winiarska K, Usarek M. DHEA supplementation to dexamethasone-treated rabbits alleviates oxidative stress in kidney-cortex and attenuates albuminuria. J Steroid Biochem Mol Biol 2017;174:17-26. 
34. Volper BD, Huynh RT, Arthur KA, Noone J, Gordon BD, Zacherle EW, et al. Influence of acute and chronic streptozotocininduced diabetes on the rat tendon extracellular matrix and mechanical properties. Am J Physiol Regul Integr Comp Physio/ 2015;309:R1135-43.

35. Morita W, Dakin SG, Snelling SJB, Carr AJ. Cytokines in tendon disease: A Systematic Review. Bone Joint Res 2017;6:656-64.

36. Amiel D, Frank C, Harwood F, Fronek J, Akeson W. Tendons and ligaments: a morphological and biochemical comparison. J Orthop Res 1984;1:257-65.

37. Aimes RT, Quigley JP. Matrix metalloproteinase-2 is an interstitial collagenase. Inhibitor-free enzyme catalyzes the cleavage of collagen fibrils and soluble native type I collagen generating the specific 3/4- and 1/4-length fragments. J Biol Chem 1995;270:5872-6.

38. Yao L, Bestwick CS, Bestwick LA, Maffulli N, Aspden RM. Phenotypic drift in human tenocyte culture. Tissue Eng 2006;12:1843-9.

39. Harada Y, Kokubu T, Mifune Y, Inui A, Sakata R, Muto T, Takase F, Kurosaka M. Dose- and timedependent effects of triamcinolone acetonide on human rotator cuff-derived cells. Bone Joint Res 2014;3:328-34.

40. Tsai WC, Liang FC, Cheng JW, Lin LP, Chang SC, Chen HH, Pang JH. High glucose concentration upregulates the expression of matrix metalloproteinase- 9 and -13 in tendon cells. BMC Musculoskelet Disord 2013;14:255.

\section{Figures}




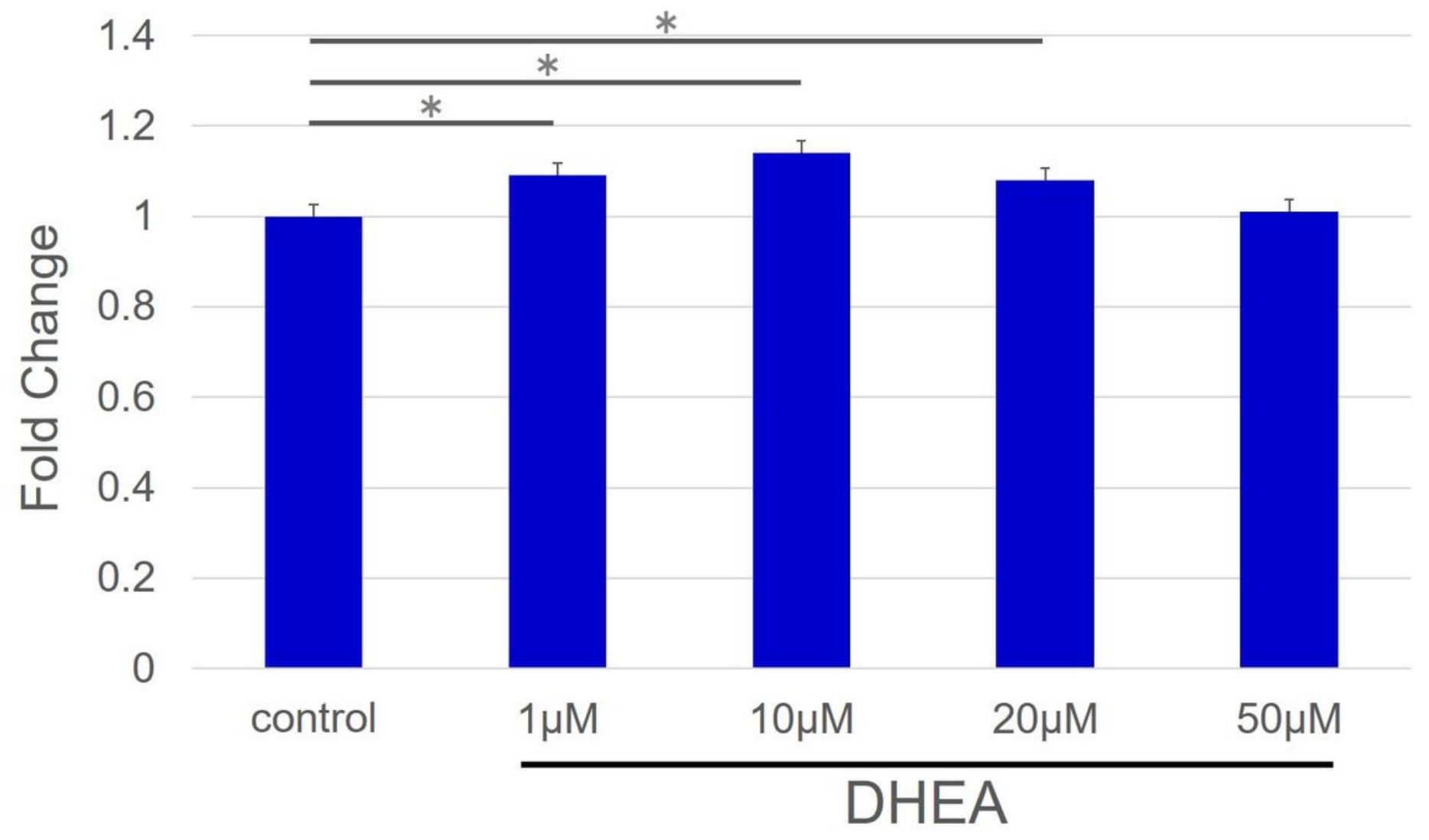

Figure 1

Cell proliferation Cell proliferation in the 1, 10, and $20 \mu \mathrm{M}$ Dehydroepiandrosterone (DHEA) groups was significantly higher than that in the control group. $(p<0.05)$. Proliferation in the 20 and $50 \mu \mathrm{M}$ DHEA groups was lower in a dose-dependent manner.
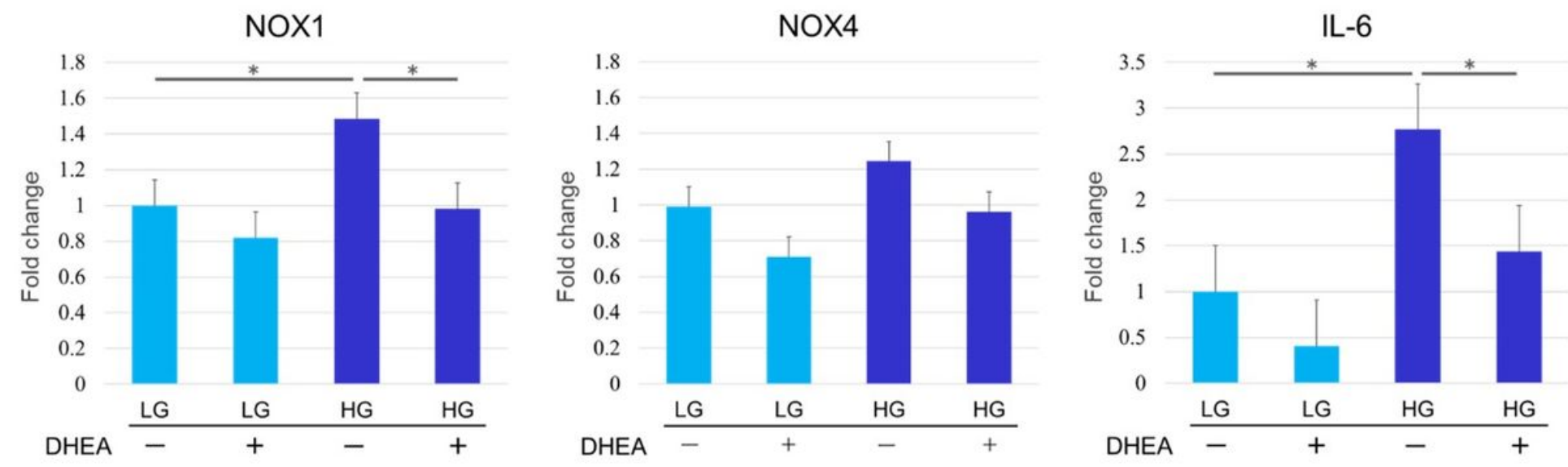

\section{Figure 2}

Quantitative real-time PCR analysis (in vitro) Messenger RNA (mRNA) expression of NADPH oxidase (NOX1) and interleukin-6 (IL-6) in the high-glucose without dehydroepiandrosterone (HG DHEA-) group were significantly higher than those in the low-glucose without DHEA (LG DHEA-) group at $48 \mathrm{hr}$. ( ${ }^{\star} \mathrm{p}<$ 
0.05). The expression of NOX1 and IL-6 in the high-glucose with DHEA group (HG DHEA+) were significantly lower than those in HG DHEA- group. ( $\left.{ }^{\star} p<0.05\right)$. There was no significant difference in NOX4 expression between the groups.

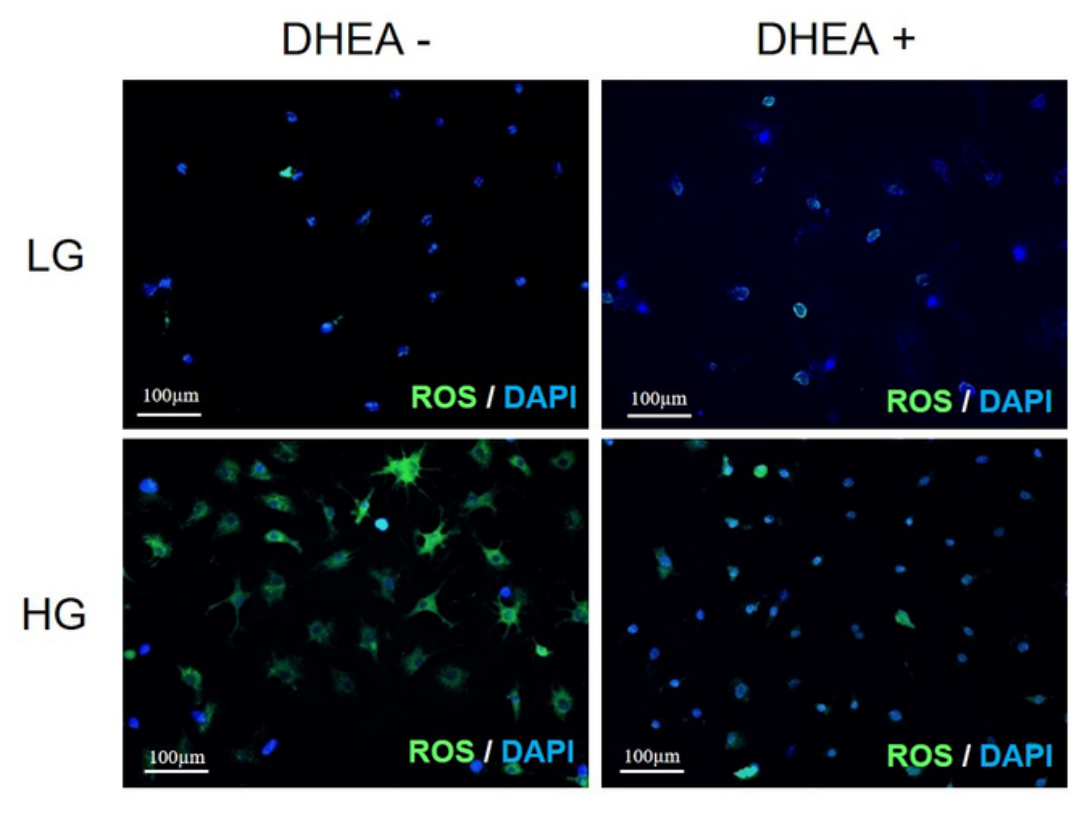

a

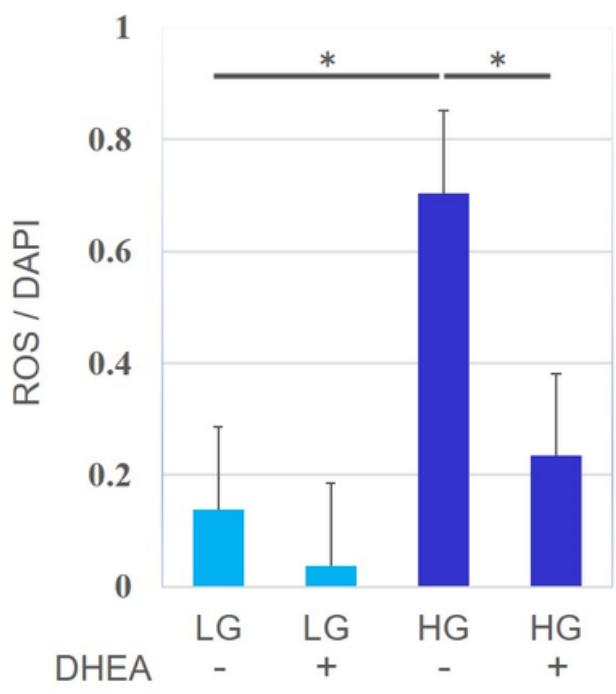

b

\section{Figure 3}

ROS accumulation (a) Fluorescence staining showing reactive oxygen species (ROS) accumulation (green) in tenocytes and nuclei (4' 6-diamidino-2-phenylindole, DAPI) (blue). There was lower ROS accumulation in the low-glucose without dehydroepiandrosterone (LG DHEA-) and with DHEA (LG DHEA+) groups. Increased ROS accumulation observed in the high-glucose (HG) groups, with the HG DHEA+ being lower than the HG DHEA- group. (b) Reactive oxygen species (ROS) accumulation was analyzed by fluorescence intensity normalized to cell number. The ROS accumulation in the high-glucose without dehydroepiandrosterone (HG DHEA-) group was greater than that in the low-glucose without DHEA (LG DHEA-) at 48 hours. The ROS accumulation in the HG DHEA+ group was significantly smaller than that in the HG DHEA- group. ${ }^{*} p<0.05$. DAPI, 4',6-diamidino-2-phenylindole. 


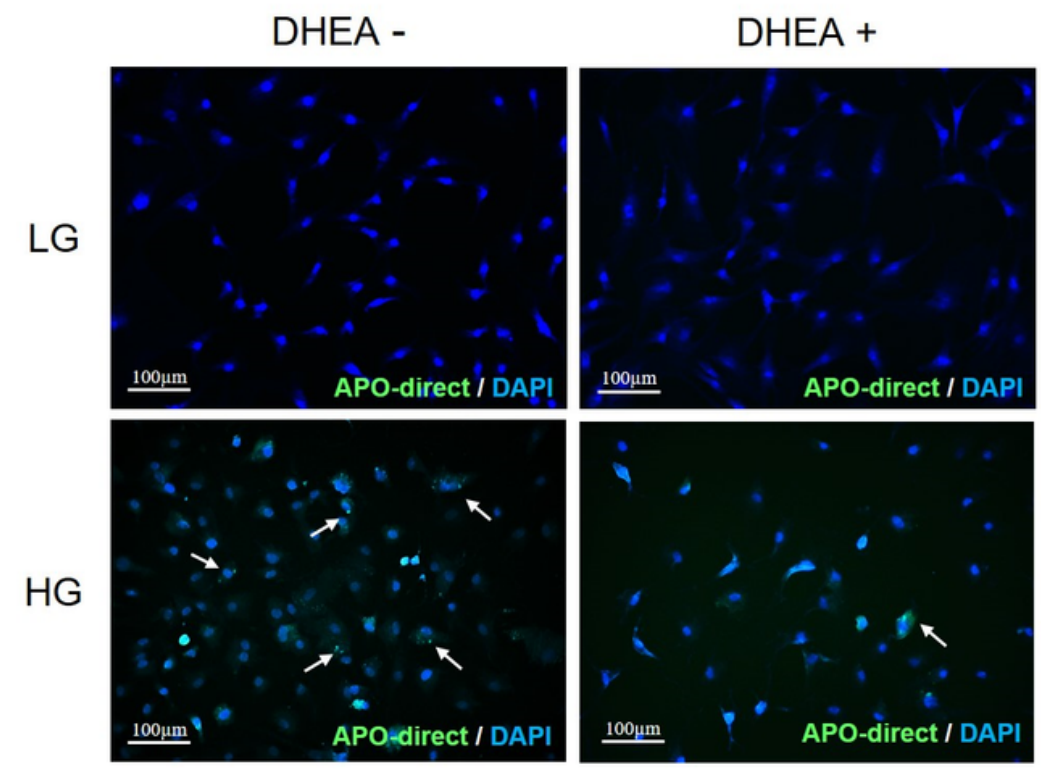

a

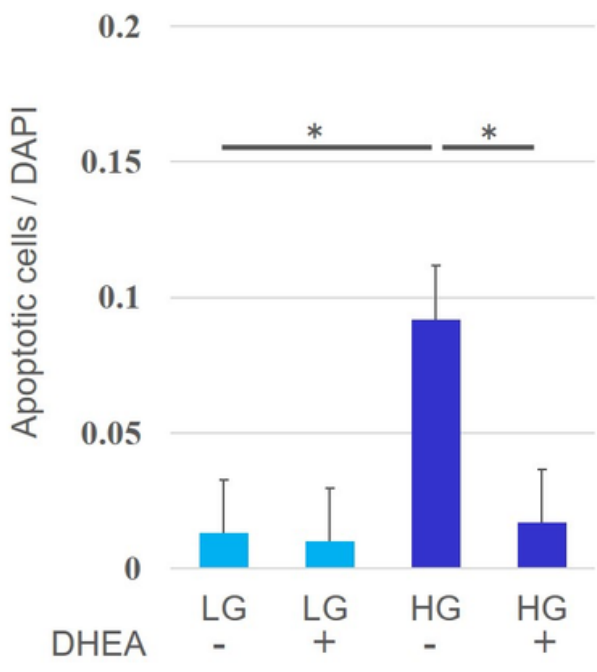

b

\section{Figure 4}

Immunofluorescence staining for analysis of apoptotic cells. (a) Immunofluorescence staining showing all cells (blue; DAPI) and apoptotic cells (green; APO-direct, white arrow $(\rightarrow)$ ). There were few apoptotic cells in the low-glucose without dehydroepiandrosterone (LG DHEA-) and with DHEA (LG DHEA+) groups. There was induction of apoptosis in the high-glucose (HG) groups. The number of apoptotic cells in the HG DHEA+ group was lower than that in the HG DHEA- group. (b) The number of apoptotic cells was analyzed by fluorescence intensity normalized to cell number. The number of apoptotic cells in the highglucose without dehydroepiandrosterone (HG DHEA-) group was significantly higher than that in lowglucose without DHEA (LG DHEA-) at 48 hours. The number of apoptotic cells in HG DHEA+ group was significantly decreased compared with that in HG DHEA- group. * $p<0.05$. DAPI, 4',6-diamidino-2phenylindole. 
Control

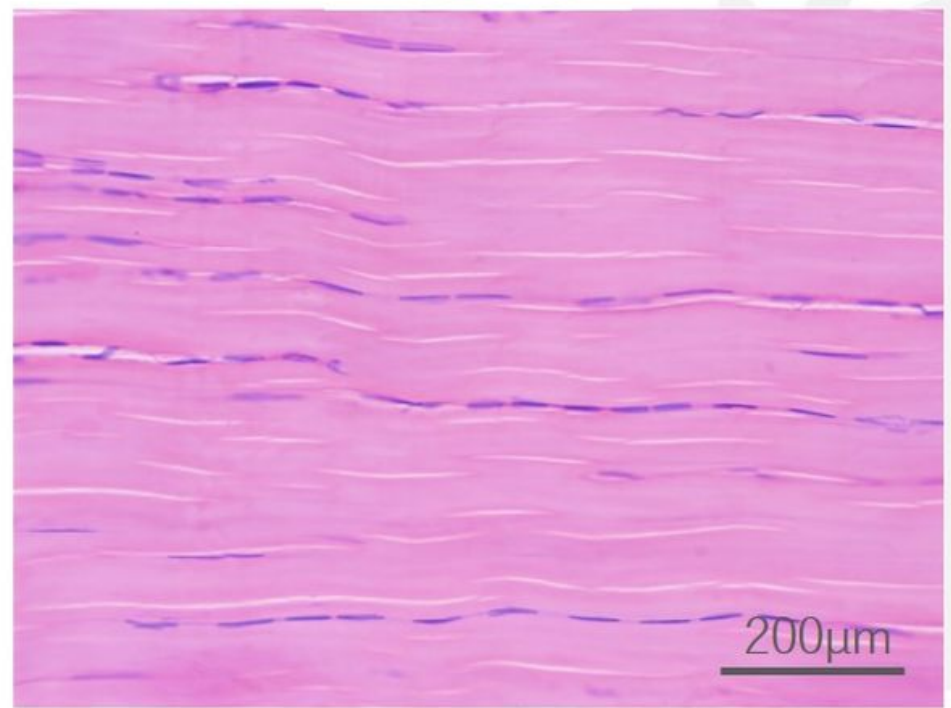

DHEA

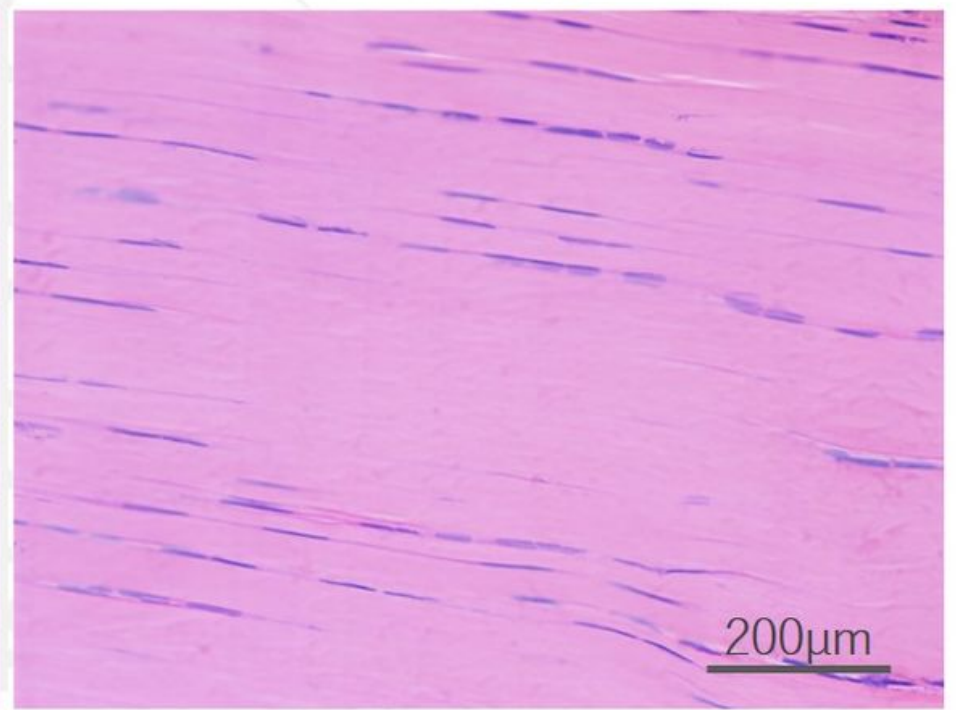

\section{Figure 5}

Hematoxylin and eosin staining of diabetic rat Achilles tendon. Hematoxylin and eosin (H\&E) staining of diabetic rat Achilles tendons in the control and dehydroepiandrosterone (DHEA) groups harvested at six weeks following streptozotocin treatment. No obvious pathological difference between groups was observed.

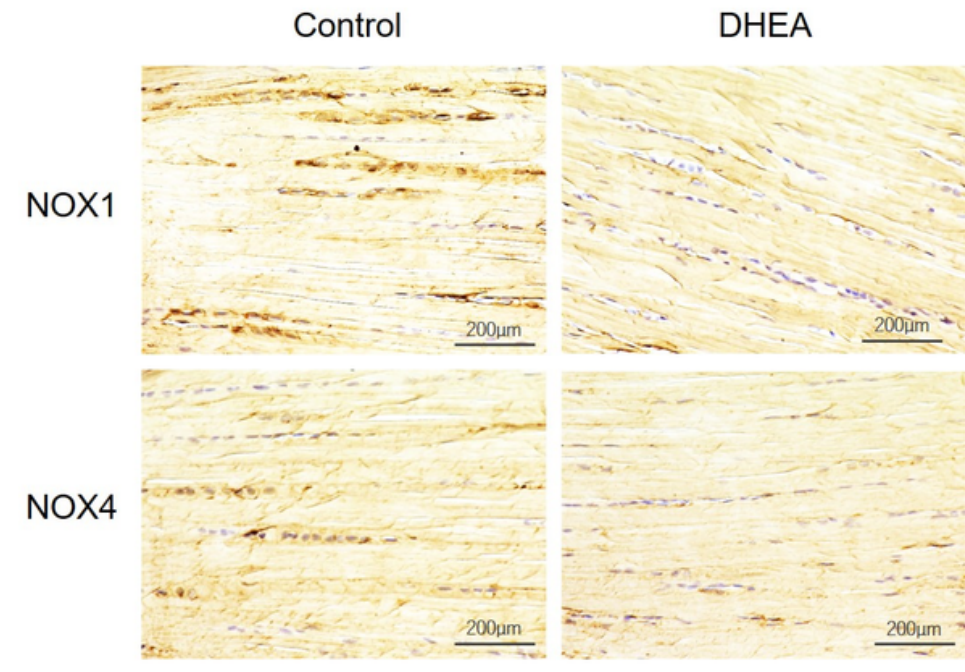

a
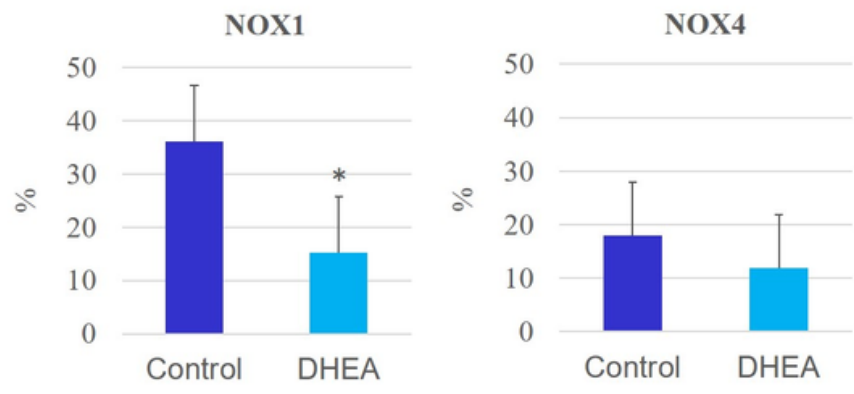

$\mathrm{b}$

\section{Figure 6}

Immunohistochemical staining for NOX1 and NOX4 expression. (a) Immunohistochemical staining for NADPH oxidase (NOX) 1 and NOX4 expression (brown) in the diabetic rat Achilles tendon. Increased expression of NOX1 in Achilles tendon was observed in dehydroepiandrosterone (DHEA) group compared with control group. There was no difference in the expression of NOX4 between DHEA and control group. 
(b) Semiquantitative analysis of cells positive for NADPH oxidase (NOX)1 (left) and NOX4 (right). The ratio of NOX1-positive cells in diabetic rat Achilles tendon was significantly higher in dehydroepiandrosterone (DHEA) group than in control group. $\left({ }^{\star} p<0.05\right)$. No significant difference was observed between control and DHEA group in the ratio of NOX4-positive cells.

\section{- Control $\quad$ DHEA}

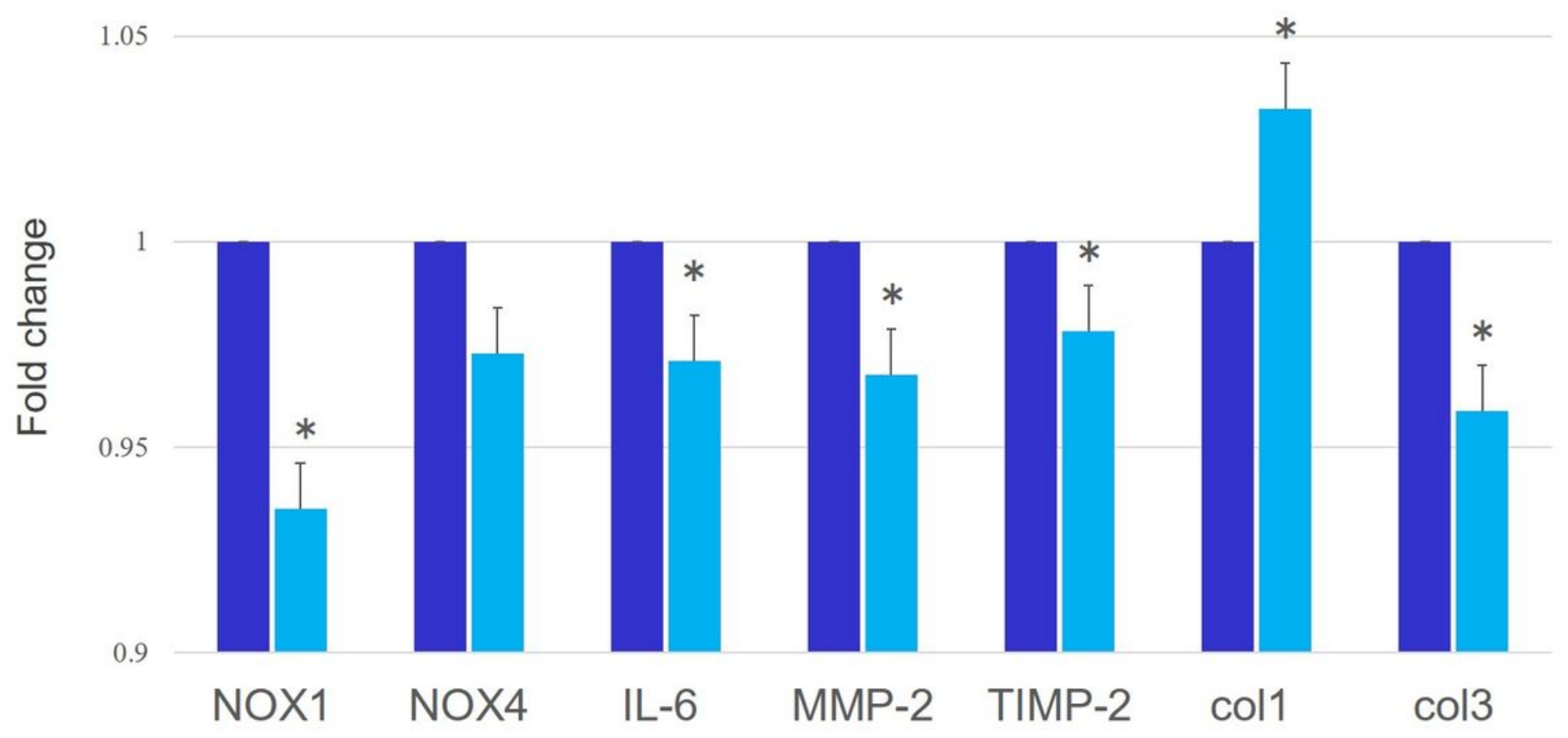

Figure 7

Quantitative real-time PCR analysis (in vivo). Relative fold changes in messenger RNA (mRNA) levels in the tendon. The mRNA expressions of NADPH oxidase (NOX)1, interleukin (IL)-6, matrix metalloproteinase (MMP)-2, tissue inhibitors of matrix metalloproteinase (TIMP)-2, and type III collagen (col3) in diabetic rat Achilles tendons were significantly lower in the dehydroepiandrosterone (DHEA) group than in the control group. ( $\left.{ }^{\star} p<0.05\right)$. No significant difference between control and diabetic rats was seen in the expression of NOX4. The expression of type I collagen (col1) was significantly higher in the DHEA group than in the control group. 